\title{
Modelos epidemiológicos fracionários: o que se perde, o que se ganha, o que se transforma?
}

\author{
Sandro Rodrigues Mazorche ${ }^{1}$ \\ DM/UFJF, Juiz de Fora, MG \\ Noemi Zeraick Monteiro ${ }^{2}$ \\ DM/UFJF, Juiz de Fora, MG
}

Resumo. Buscamos investigar o uso de derivadas fracionárias no modelo SIR, tanto analiticamente quanto por meio de simulações. Nos interessamos pelos questionamentos de persistência de características na transição do modelo inteiro para o fracionário. Em particular, analisamos unidades, conservação da população, a impossibilidade de utilizar a derivada de Riemann-Liouville, cuidados com a não negatividade e a monotonicidade e, finalmente, o ponto de pico e os equilíbrios.

Palavras-chave. Modelo SIR, Derivadas Fracionárias, Persistência de Características

\section{Introdução}

A matemática está desempenhando um papel cada vez mais importante na física e nas ciências biológicas, fazendo ressurgir o interesse por técnicas modernas e clássicas da matemática aplicada [4]. A biologia se torna mais quantitativa e, por um lado, temos a realidade física, fornecendo dados. Por outro, a elaboração de hipóteses e teorias que buscam organizar a realidade por meio de leis matemáticas: é a construção de um modelo. O modelo criado, então, é estudado matematicamente e computacionalmente, após o que necessita ser confrontado com os dados para sua validação. Assim, a teoria matemática e a realidade se retroalimentam de maneira contínua.

A modelagem fracionária permite capturar a dependência de estágios anteriores em materiais ou processos e, nesse contexto, torna mais próximos da realidade fenômenos biológicos, reológicos, sistemas mecânicos, elétricos etc [22]. Comumente, um modelo já existente é flexibilizado pela substituição da ordem inteira da derivada por uma fracionária. Em particular, modelos tipo SIR têm sido largamente estudados com ordens fracionárias (exemplos são citados ao longo do texto).

Embora essa substituição possa produzir estimativas muito acuradas, questionamos: quais características do modelo original são mantidas? A mudança na ordem das derivadas estabelece automaticamente modelos consistentes quanto à definição de parâmetros, significado físico, conservação e unidades? O que dizer sobre não negatividade, monotonicidade (quando houver), entre outras questões? O uso de técnicas para resolver esses modelos analiticamente ou numericamente é um campo interessante por si só. Porém, do ponto de vista da modelagem, é importante tentar verificar como, onde e por que as derivadas fracionárias interferem no modelo. Esses questionamentos são trabalhados na Seção 3, após alguns preliminares. Na Seção 4, comparamos teoria e resultados numéricos e, na Conclusão, citamos um exemplo diferenciado de modelagem fracionária.

\section{Preliminares}

Nesta seção, apresentamos as principais definições e resultados utilizados no trabalho.

\subsection{O Cálculo Fracionário}

Em uma carta de 1695, l'Hôpital questionou Leibniz sobre a possibilidade e o significado de uma derivada de ordem $1 / 2$, acontecimento que pode ser considerado como o nascimento do Cálculo Fracionário. Nos três séculos seguintes, importantes avanços foram realizados por Liouville, Riemann,

\footnotetext{
${ }^{1}$ sandro.mazorche@ufjf.edu.br.

${ }^{2}$ nzmonteiro@ice.ufjf.br.
} 
Grünwald, Caputo e muitos outros. Porém, somente após a primeira Conferência Internacional sobre Cálculo Fracionário e Aplicações, em 1974, o número de pesquisadores do Cálculo Fracionário apresentou grande crescimento, sendo inclusive propostas novas formulações. Atualmente, congressos e simpósios ocorrem com mais frequência e regularidade, tendo sido realizada em 2020 a Primeira Conferência Online sobre Cálculo Fracionário Moderno e suas Aplicações (OCMFCA2020). O leitor poderá consultar a referência [19] para uma cronologia resumida de publicações em Cálculo Fracionário no período de 1695 a 2019, assim como para definições e resultados gerais.

Abaixo, consideramos $[a, b] \subset \mathbb{R}, \alpha \in \mathbb{R}_{+}^{*}$, uma função $f \in L^{p}[a, b], p \geq 1$, e $n-1<\alpha<n$, com $n \in \mathbb{N}$. A derivada de ordem inteira $n$ é representada por $D^{n}$ e $\Gamma$ é a função gama.

Definição 2.1. A integral fracionária de Riemann-Liouville de ordem $\alpha$ é definida para $t \in[a, b]$ por

$$
{ }_{a} J_{t}^{\alpha} f(t)=\frac{1}{\Gamma(\alpha)} \int_{a}^{t}(t-\theta)^{\alpha-1} f(\theta) d \theta
$$

Definição 2.2. A derivada fracionária de Riemann-Liouville de ordem $\alpha$ é definida para $t \in[a, b]$ por

$$
{ }_{a}^{R-L} D_{t}^{\alpha} f(t)=\frac{1}{\Gamma(n-\alpha)}\left(\frac{d^{n}}{d t^{n}}\right) \int_{a}^{t}(t-\theta)^{n-\alpha-1} f(\theta) d \theta .
$$

Em outras palavras,

$$
{ }_{a}^{R-L} D_{t}^{\alpha} f(t)=D^{n}\left[{ }_{a} J_{t}^{n-\alpha} f(t)\right] .
$$

Definição 2.3. A derivada fracionária de Caputo de ordem $\alpha$ é definida para $t \in[a, b]$ por

$$
{ }_{a}^{C} D_{t}^{\alpha} f(t)=\frac{1}{\Gamma(n-\alpha)} \int_{a}^{t}(t-\theta)^{n-\alpha-1} \frac{d^{n}}{d \theta^{n}} f(\theta) d \theta .
$$

Em outras palavras,

$$
{ }_{a}^{C} D_{t}^{\alpha} f(t)={ }_{a} J_{t}^{n-\alpha}\left[D^{n} f(t)\right] .
$$

Se $f$ é contínua, a integral fracionária de Riemann-Liouville é o inverso à direita tanto da derivada fracionária de Riemann-Liouville quanto daquela de Caputo [21], isto é, ${ }_{a}^{C} D_{t}^{\alpha}{ }_{a} J_{t}^{\alpha} f(t)=$ ${ }_{a}^{R-L} D_{t \quad a}^{\alpha} J_{t}^{\alpha} f(t)=f(t)$. Utilizamos ainda, neste trabalho, o seguinte resultado:

Proposição 2.1. A transformada de Laplace da derivada fracionária de Caputo é dada por [19]:

$$
\mathcal{L}\left[{ }_{0}^{C} D_{t}^{\alpha} f(t)\right](s)=s^{\alpha} \mathcal{L}[f(t)](s)-\sum_{k=0}^{n-1} s^{\alpha-1-k} f^{(k)}(0) .
$$

Observação 2.1. Em (6), as condições iniciais envolvem derivadas de ordem inteira em um ponto. Além disso, a derivada de Caputo de uma constante é zero, o que pode favorecer sua utilização. Quanto à redução à ordem inteira, as derivadas fracionárias de Riemann-Liouville e Caputo possuem compatibilidade reversa, isto é, $\lim _{\alpha \rightarrow n}{ }_{a}^{C} D_{t}^{\alpha} f(t)=\lim _{\alpha \rightarrow n}{ }_{a}^{R-L} D_{t}^{\alpha} f(t)=D_{t}^{n} f(t)$ [21]. Mas, enquanto $\lim _{\alpha \rightarrow n-1}{ }^{R-L} D_{t}^{\alpha} f(t)=D^{n-1} f(t)$, temos $\lim _{\alpha \rightarrow n-1}{ }_{a}^{C} D_{t}^{\alpha} f(t)=D^{n-1} f(t)-D^{n-1} f(a)$.

Finalmente, ressaltamos que resultados sobre monotonicidade de funções com derivadas fracionárias positivas ou negativas não são triviais como no caso inteiro. Não obstante, valem específicos Teoremas do Valor Médio [13].

\subsection{O Modelo SIR}

Em 1927, o modelo SIR (Suscetíveis-Infectados-Removidos) foi introduzido de maneira notável em um trabalho proposto por Kermack e McKendrick [16]. O formalismo matemático que embasou cuidadosamente a construção desse modelo epidemiológico é, infelizmente, pouco difundido, sendo apresentada, em geral, a versão "lapidada" do modelo, sintetizada na Figura 1. 


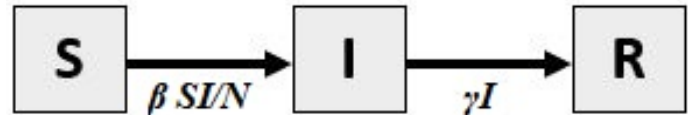

Figura 1: Diagrama de fluxo do modelo SIR sem dinâmica vital.

$$
\begin{gathered}
S^{\prime}(t)=-\beta S(t) I(t) / N, \\
I^{\prime}(t)=\beta S(t) I(t) / N-\gamma I(t), \\
R^{\prime}(t)=\gamma I(t) .
\end{gathered}
$$

No diagrama, $S(t)$ é a quantidade de suscetíveis, $I(t)$ a quantidade de infectados e $R(t)$ a quantidade de removidos, por recuperação ou morte, no tempo $t$. A população $N=S(t)+$ $I(t)+R(t)$ é constante e admite-se que os recuperados estão imunes durante o período em análise. Também ignoramos o período de latência da doença. O modelo é escrito como em (7)-(9), onde $\beta$ é o coeficiente de transmissão, relacionado, por exemplo, à quantidade de contatos de um indivíduo e à probabilidade de que um contato resulte em contágio, e $\gamma$ é a taxa na qual os indíviduos passam do compartimento infeccioso para o compartimento dos removidos, ambos com unidade [tempo] ${ }^{-1}$.

Ao longo do desenvolvimento da epidemiologia matemática, os modelos compartimentais sofreram extensões de modo que sua estrutura se adequasse a diferentes tipos de doenças. Além disso, há doenças transmitidas por vetores e cada modelo pode ser tratado com diferentes hipóteses, como a consideração ou não da dinâmica vital, a possibilidade de vacinação etc. Para um aprofundamento, sugerimos a referência [4] e outros trabalhos dos autores, assim como as demais referências.

\subsubsection{O número de reprodução}

O número de reprodução $\Re$ reflete o quão infecciosa é uma doença num dado contexto, enquanto o número básico de reprodução, $\Re_{0}$, é o número de reprodução quando não há imunidade nem intervenção na transmissão da doença. Em condições de homogeneidade da população, $\Re$ é definido como o número médio de infecções que um único indivíduo pode gerar durante seu período infeccioso [15]. O $\Re_{0}$ representa este número no início da infecção, ou seja, o número médio de infecções que um indivíduo pode gerar após ser introduzido em uma população completamente suscetível.

Em modelos tradicionais, as epidemias não podem ocorrer quando o $\Re_{0}$ é inferior a 1 e surtos estabelecidos desaparecerão se as intervenções ou o esgotamento da parte suscetível da população forem suficientes para manter $\Re$ abaixo de 1 . Nos modelos simples, como no SIR clássico, temos $\Re(t)=\Re_{0} S(t) / N$. Assim, o comportamento da infecção é quase completamente determinado pelo $\Re_{0}$, cujo valor determina não apenas a mudança da estabilidade local do equilíbrio livre de doença, mas também quando o equilíbrio endêmico se torna possível [15].

\section{Modelo SIR Fracionário}

Como mencionado anteriormente, a modelagem fracionária, em geral, corresponde a trabalhar as EDO's clássicas substituindo a derivada inteira por uma fracionária. Esses modelos podem produzir estimativas muito boas, mas é preciso cuidado com a persistência de propriedades na transição de modelos de derivada inteira para seus correspondentes fracionários: segundo a construção feita por Kermack e McKendrick, em consistência aos dados reais, deveríamos ter parâmetros definidos biologicamente com unidades corretas, população constante (pois não há dinâmica), não negatividade do modelo e, novamente na ausência de dinâmica, monotonicidade para os compartimentos $S$ e $R$, pois o fluxo é criado de $S$ para $I$ e de $I$ para $R$. Além disso, o pico da infecção, e consequente início de seu declínio, ocorre classicamente quando $\Re(t)=1$. Essas características são mantidas?

Inicialmente, analisamos a persistência da população total. O artigo [9] demonstra, em um sistema simples de dois compartimentos, que a utilização de ordens diferentes em cada compartimento leva à violação do equilíbrio de massa. Os autores defendem, num artigo do mesmo ano [10], a argumentação de que, tanto no contexto da farmacocinética então considerado quanto em sistemas compartimentais análogos, um fluxo de massa de saída definido como uma taxa de ordem 
fracionária não pode aparecer como um fluxo de entrada em outro compartimento, com uma ordem fracionária diferente, sem violar o balanço de massa. Analisamos a relação entre as ordens e a população total $N=S+I+R$ do modelo SIR fracionário, onde $N_{0}=S_{0}+I_{0}+R_{0}$ :

$$
\begin{gathered}
D^{\alpha_{1}} S(t)=-\beta_{1} S(t) I(t) / N_{0}, \\
D^{\alpha_{2}} I(t)=\beta_{2} S(t) I(t) / N_{0}-\gamma_{2} I(t), \\
D^{\alpha_{3}} R(t)=\gamma_{3} I(t) .
\end{gathered}
$$

Começamos com a derivada de Caputo e $\alpha_{1}, \alpha_{2}, \alpha_{3} \in(0,1]$ (ex.: [1]; [11]). Aplicando a transformada de Laplace e realizando operações nas equações (10) - (12), obtemos

$$
\mathcal{L}\{S+I+R\}=\frac{S_{0}+I_{0}+R_{0}}{s}+\left[\frac{\beta_{2}}{N_{0} s^{\alpha_{2}}}-\frac{\beta_{1}}{N_{0} s^{\alpha_{1}}}\right] \mathcal{L}\{S I\}+\left[\frac{\gamma_{3}}{s^{\alpha_{3}}}-\frac{\gamma_{2}}{s^{\alpha_{2}}}\right] \mathcal{L}\{I\} .
$$

Aplicando a transformação inversa e as convoluções, finalmente escrevemos

$$
N=N_{0}+\int_{0}^{t}\left[\frac{\beta_{2}(t-\theta)^{\alpha_{2}-1}}{N_{0} \Gamma\left(\alpha_{2}\right)}-\frac{\beta_{1}(t-\theta)^{\alpha_{1}-1}}{N_{0} \Gamma\left(\alpha_{1}\right)}\right] S(\theta) I(\theta) d \theta+\int_{0}^{t}\left[\frac{\gamma_{3}(t-\theta)^{\alpha_{3}-1}}{\Gamma\left(\alpha_{3}\right)}-\frac{\gamma_{2}(t-\theta)^{\alpha_{2}-1}}{\Gamma\left(\alpha_{2}\right)}\right] I(\theta) d \theta .
$$

Assim, para que $N$ seja constante, devemos ter $\alpha_{1}=\alpha_{2}=\alpha_{3}$ e os parâmetros devem satisfazer $\beta_{1}=\beta_{2}$ e $\gamma_{2}=\gamma_{3}$, independentemente da maneira na qual eles foram definidos. O grande problema não é modificar $N$, o que pode ocorrer por dinâmica vital, migração etc., mas justificar a mudança. Neste caso, sem migração ou dinâmica vital, as pessoas não poderiam sumir ou aparecer.

Aqui, é importante notar que não poderíamos utilizar a definição de Riemann-Liouville sequer mantendo as mesmas ordens em cada compartimento. Com efeito, teríamos ${ }^{R-L} D^{\alpha} N(t)=0$, mas a derivada de Riemann-Liouville de uma constante não nula não é zero, ou seja, novamente haveria problemas com a população total. Além disso, as condições iniciais para a derivada de RiemannLiouville não são facilmente interpretadas. Assim, doravante utilizamos a derivada de Caputo e ordens iguais, passando a analisar o balanceamento das unidades. O tempo 0 é considerado o estágio inicial da doença e tomamos condições iniciais iguais às do modelo de ordem inteira.

Partindo do modelo (7)-(9) ou de suas diversas extensões, alguns autores (ex.: [14]; [17]) substituem a ordem da derivada mantendo os mesmos parâmetros $\beta$ e $\gamma$, obtendo (15)-(17).

$$
\begin{array}{ccc}
D^{\alpha} S(t)=-\beta S(t) I(t) / N, & (15) & D^{\alpha} S(t)=-\beta^{\alpha} S(t) I(t) / N, \\
D^{\alpha} I(t)=\beta S(t) I(t) / N-\gamma I(t), & (16) & D^{\alpha} I(t)=\beta^{\alpha} S(t) I(t) / N-\gamma^{\alpha} I(t), \\
D^{\alpha} R(t)=\gamma I(t), & (17) & D^{\alpha} R(t)=\gamma^{\alpha} I(t) .
\end{array}
$$

Observamos que o lado esquerdo do sistema tem unidade [tempo $]^{-\alpha}$. Portanto, o lado direito deve possuir esta unidade. Apenas abolir as unidades não resolve o problema. Segundo [9], as unidades, na verdade, ajudam a revelar a questão: uma taxa de ordem 1, por exemplo, é um tipo diferente de taxa daquela de ordem $1 / 2$, da mesma maneira que, na física clássica, a taxa de ordem 1, velocidade, é diferente daquela correspondente à ordem 2, a aceleração, não apenas pela diferença de unidades. Outros autores (ex.: [2]; [12]) corrigem a dimensão multiplicando-as por constantes de tempo ou elevando os parâmetros a $\alpha$. Nesse último caso, o modelo SIR fracionário seria escrito da forma (18)-(20). As unidades estão balanceadas, mas não obtivemos significado físico para esses tipos de correção dimensional.

Agora, para um sistema sem dinâmica vital, é esperado que os compartimentos $S$ e $R$ sejam monótonos não crescente e não decrescente, respectivamente. Além disso, deseja-se que a solução seja não negativa para todo $t \in[0, \infty)$. Com o objetivo de provar alguma dessas afirmações ou ambas, muitos autores (ex.: [2]; [12]) utilizam o seguinte lema:

Lema 3.1. Seja $\alpha \in(0,1)$ e suponha $f(t), D^{\alpha} f(t) \in C[0, b]$. Segue do Teorema do Valor Médio $q u e$, se $D^{\alpha} f(t) \geq 0$ no intervalo $[0, b]$, então $f$ é não decrescente em $[0, b]$. Analogamente, se $D^{\alpha} f(t) \leq 0$ no intervalo $[0, b]$, então $f$ é não crescente neste intervalo. 
Porém, sem outras hipóteses esse resultado não é válido. As referências [6] e [7] mostram que o sinal da derivada fracionária não é suficiente, por si só, para resultados de monotonicidade. Acreditamos que a não negatividade possa ser obtida da propriedade de que duas soluções não podem se cruzar, como provado para uma dimensão em [8]. Contudo, as simulações parecem indicar que, no modelo fracionário sem dinâmica vital, os compartimentos $S$ e $R$ podem oscilar.

Finalmente, no modelo original, o pico da infecção ocorre quando $S(t)=\gamma N / \beta$ ou, equivalentemente, $\Re(t)=1$. No caso geral, essa característica pode não ser mantida:

Teorema 3.1. Se uma função $f \in C^{1}[0, b]$ admite um máximo (mínimo) global em $t \in(0, b)$, então $D^{\alpha} f(t) \geq 0(\leq 0)$, para todo $\alpha \in(0,1)[7]$.

Quanto aos equilíbrios, sem dinâmica vital, todos os pontos do conjunto $\{(S, 0, R) ; S, R \geq$ $0, S+R=N\}$ são equilíbrios. O equilíbrio estável $(S, I, R)_{\infty}$ para o caso $\alpha=1$ é calculado em [16]. Contudo, há passagens não válidas para derivadas fracionárias e, embora os pontos de equilíbrio sejam os mesmos, as regiões de estabilidade podem mudar, como ilustrado na Seção 4.

\section{Resultados Numéricos}

Procuramos corroborar as discussões realizadas implementando no MATLAB o modelo SIR fracionário geral (10)-(12), com código próprio baseado em esquema tipo L1 [20]. Os resultados numéricos permitem observar diversas características que podem não persistir ao mudarmos a ordem da derivada, como a população total, a monotonicidade, a região de estabilidade do equilíbrio $\left(S_{\infty}, I_{\infty}\right)$ e a condição do ponto de pico. Utilizamos uma população $N=1000000$, condições iniciais $S(0)=N-1, I(0)=1$ e $R(0)=0$, parâmetros $\beta=1, \gamma=0.3$ e $d t=0.1$. Na Figura 2, temos o modelo de ordem inteira. Notamos a monotonicidade dos compartimentos $S$ e $R$ e a constância da população total. Na Figura 3, utilizando a derivada de Caputo e ordens diferentes, observamos que a população total não é mantida. Lembramos que a população não se mantém com o modelo em derivada de Riemann-Liouville mesmo com ordens iguais, conforme Figura 4. Nas demais figuras, utilizamos a derivada de Caputo. Apenas corrigir as unidades, como elevar os parâmetros a $\alpha$, não modifica algumas características observadas, como a não monotonicidade. Com efeito, na Figura 5 , consideramos $\beta_{1}=\beta_{2}=\beta$ e $\gamma_{2}=\gamma_{3}=\gamma$ e, na Figura 6 , tomamos $\beta_{1}=\beta_{2}=\beta^{\alpha}$ e $\gamma_{2}=\gamma_{3}=\gamma^{\alpha}$. Verificamos que $S$ e $R$ não são monótonos, ou seja, os fluxos não podem ser considerados da maneira tradicional: há, por exemplo, uma reentrada no compartimento $S$. Acreditamos que isso não é corrigido ao ajustar as unidades: a natureza das taxas foi modificada. Finalmente, na Figura 7, exibimos o plano $(S, I)$ para diversas ordens $\alpha$ e as mesmas condições iniciais. A trajetória externa equivale ao modelo tradicional, o ponto rosa indica $S=\gamma N / \beta$ e, a seta, o sentido de crescimento de $\alpha$. O tempo máximo foi de $T=10000$. Consideramos os mesmos parâmetros já que, nessa figura, um de nossos interesses é investigar os pontos de equilíbrio e o valor de $S$ no pico.

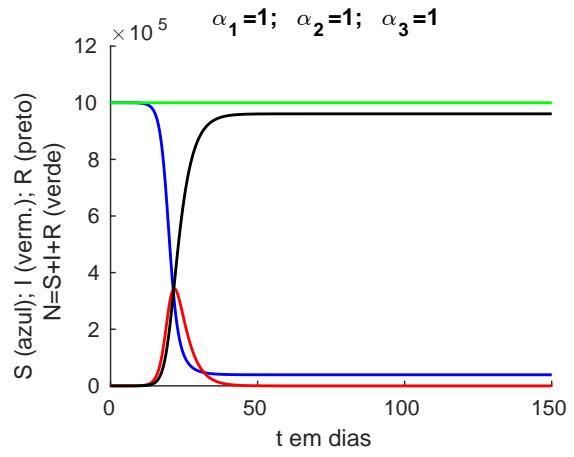

Figura 2: Modelo SIR Clássico.

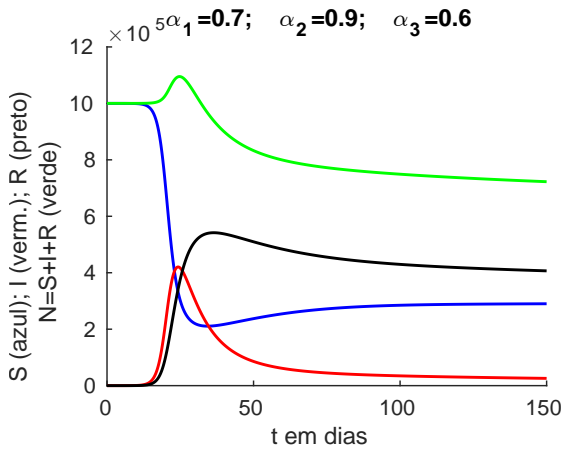

Figura 3: Variação da população (C). 


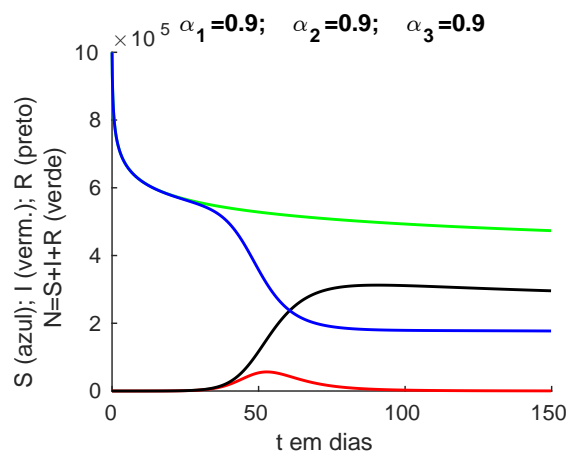

Figura 4: Variação da população (R-L).

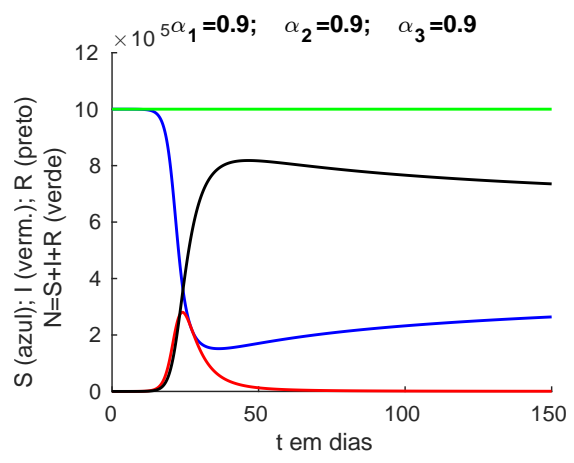

Figura 6: Parâmetros elevados a $\alpha$.

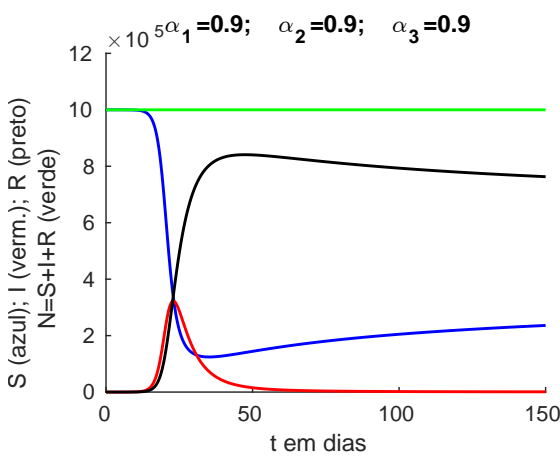

Figura 5: Parâmetros não elevados a $\alpha$.

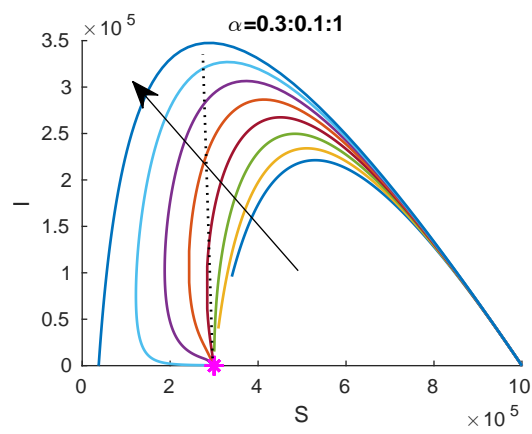

Figura 7: Plano $(S, I)$.

\section{Conclusões}

O presente trabalho não pretende invalidar os diversos modelos fracionários desenvolvidos nos últimos anos. Inclusive, sugerimos a leitura de [5] para um estudo inovador, por meio de uma abordagem estatística, do efeito memória tratado pelo Cálculo Fracionário. Na verdade, é natural que novos modelos não herdem todas as características do original; porém, é preciso estarmos atentos ao que mudou, pois é interessante não apenas aproximar bem as curvais reais, mas entender do ponto de vista físico/biológico o que está acontecendo com as taxas e parâmetros. Até o presente, não conseguimos uma construção física que permita trocar as ordens das derivadas clássicas, ainda que as unidades sejam corrigidas e que as novas ordens sejam iguais em todos os compartimentos. Ainda, não há garantia de monotonicidade pois, em particular, o sinal da derivada fracionária deixa de valer como indicativo do (de)crescimento da função em dado intervalo.

Finalizando, acreditamos que é possível modelar um sistema fisicamente, com formalismo semelhante ao dos "pais" Kermack e McKendrick, de maneira que o surgimento de derivadas fracionárias decorra de leis potenciais nas funções de infecciosidade e remoção. Assim, em [18], apresentamos uma derivação física de um modelo fracionário, seguindo os passos de Angstmann, Henry e McGann [3] e a linguagem probabilística dos Passeios Aleatórios em Tempo Contínuo (PATC). Ainda em [18], modelamos a COVID-19 em seus meses iniciais. Trabalhos futuros pretendem fornecer análises de parâmetros e um maior entendimento do problema de valor inicial e dos pontos de equilíbrio tanto do modelo fracionário discutido quanto do modelo apresentado em [18].

\section{Agradecimentos}

Agradecemos o apoio da Coordenação de Aperfeiçoamento de Pessoal de Nível Superior - Brasil (CAPES) - Código de Financiamento 001. 


\section{Referências}

[1] Ahmad, A., Farman, M., Ahmad, M. O., Raza, N. and Abdullah, M. Dynamical behavior of SIR epidemic model with non-integer time fractional derivatives: a mathematical analysis, Int. J. Adv. Appl. Sci., 5:123-129, 2018. DOI: 10.21833/ijaas.2018.01.016.

[2] Almeida, R. Dynamical analysis of a fractional SIR model with treatment and quarantine, Chaotic Modeling and Simulation, 2:115-124, 2020.

[3] Angstmann, C. N., Henry, B. I. and McGann, A. V. A fractional-order infectivity and recovery SIR model, Fractal and Fractional, 1:11, 2017. DOI: 10.3390/fractalfract1010011.

[4] Brauer, F. and Castillo-Chavez, C. Mathematical models in population biology and epidemiology. Vol. 2. Springer, New York, 2012.

[5] Barros, L.C., Lopes, M. M., Simões, F. S. P., Esmi, E., Santos, J.P.C. dos, and Sánchez, D. E. The memory effect on fractional calculus: an application in the spread of Covid-19, Computational and Applied Mathematics, 40:72, 2021. DOI: 10.21203/rs.3.rs-41084/v1.

[6] Choi, S. K. and Koo, N. The monotonic property and stability of solutions of fractional differential equations, Nonlinear Analysis: Theory, Methods and Applications, 74:6530-6536, 2011. DOI: 10.1016/j.na.2011.06.037.

[7] Diethelm, K. Monotonicity of functions and sign changes of their Caputo derivatives, Fractional Calculus and Applied Analysis, 19:561-566, 2016. DOI: 10.1515/fca-2016-0029.

[8] Diethelm, K. The analysis of fractional differential equations: an application-oriented exposition using differential operators of Caputo type. Springer Science \& Business Media, Berlin, 2010.

[9] Dokoumetzidis, A., Magin, R. and Macheras, P. A commentary on fractionalization of multicompartmental models, Journal of Pharmacokinetics and Pharmacodynamics, 37:203-207, 2010. DOI: 10.1007/s10928-010-9153-5.

[10] Dokoumetzidis, A., Magin, R. and Macheras, P. Fractional kinetics in multi-compartmental systems, Journal of Pharmacokinetics and Pharmacodynamics, 37:507-524, 2010. DOI: 10.1007/s10928-0109153-5.

[11] Farman, M., Ahmad, A., Muslim, H. and Ahmad, M. O. Dynamical behavior of Hepatitis B fractionalorder model with modeling and simulation, Journal of Biochemical Technology, 10:11-17, 2019.

[12] González-Parra, G., Arenas, A. J. and Chen-Charpentier, B. M. A fractional order epidemic model for the simulation of outbreaks of influenza A (H1N1), Mathematical Methods in the Applied Sciences, 37:2218-2226, 2013. DOI: 10.1002/mma.2968.

[13] Guo P., Changpin L. and Guanrong C. On the fractional mean-value theorem, International Journal of Bifurcation and Chaos, 22:1250104(1-6), 2012. DOI: 10.1142/S0218127412501040.

[14] Hasan, S., Al-Zoubi, A., Freihet, A., Al-Smadi, M. and Momani, S. Solution of fractional SIR epidemic model using residual power series method, Appl. Math. Inf. Sci, 13:153-161, 2019. DOI: $10.18576 /$ amis/130202.

[15] Hethcote, H. W. The mathematics of infectious diseases, SIAM review, 42:599-653, 2000. DOI: $10.1137 / \mathrm{S} 0036144500371907$.

[16] Kermack, W. O. and McKendrick, A. G. Contributions to the mathematical theory of epidemics-I (1927), Bulletin of Mathematical Biology, 53:33-55, 1991. DOI: 10.1007/BF02464423.

[17] Khan, M.A. and Atangana, A. Modeling the dynamics of novel coronavirus (2019-nCov) with fractional derivative, Alexandria Engineering Journal, 59:2379-2389, 2020. DOI: 10.1016/j.aej.2020.02.033.

[18] Monteiro, N. Z. and Mazorche, S. R. Fractional derivatives applied to epidemiology, Trends in Computational and Applied Mathematics, 22:157-177, 2021. DOI: 10.5540/tcam.2021.022.02.00157.

[19] Oliveira, E. C de. Solved exercises in fractional calculus. Springer International Publishing, Cham, 2019.

[20] Oldham, K. and Spanier, J.The fractional calculus theory and applications of differentiation and integration to arbitrary order. Academic Press, New York, 1974.

[21] Ortigueira, M. D. and Machado, J. A. T. What is a fractional derivative?, Journal of Computational Physics, 293:4-13, 2015. DOI: 10.1016/j.jcp.2014.07.019.

[22] Podlubny, I. Fractional differential equations: an introduction to fractional derivatives. Academic Press, San Diego, 1999. 